\title{
Modeling and optimization of open crack detection by active thermography
}

\author{
by A. Thiam*, J.C. Kneip*, S. Mattei*, E. Cicala* \\ *Equipe LTm, axe PMDM, laboratoire Interdisciplinaire Carnot de Bourgogne UMR 6303 CNRS - \\ Université de Bourgogne Franche-Comté, 1 Allée des Granges Forestier, 71100 Chalon/Saône, France, \\ abdoulahad.thiam@u-bourgogne.fr
}

\begin{abstract}
The aim of the presented work is the detection of open defects in metallic materials using laser-material interaction coupled with infrared thermal images. This process is a possible alternative for magnetic particles testing and penetrant testing in the field of non-destructive testing. In this context, a numerical simulation model is implemented with Comsol Multiphysics $\AA^{\circ}$. This model allows us to look for the process optimal parameters through numerical experimental designs.
\end{abstract}

\section{Introduction}

Nowadays non-destructive testing becomes essential in industrial environment, as well at the time of the manufacturing of materials or structures, as on installation in service in order to verify their health status. Among all available techniques penetrate testing [1] and magnetic particles testing [2] are massively used for surface anomalies. On-site, inspections are manually realized, on limited dimensions zones, and require use of revealing products. For several years, on specific application, companies are in search of alternative methods. Among several techniques, active thermography [3] appears as an interesting possibility.

Generally, thermographic methods used on metallic materials are based on the "flying spot". The first developments go back to 1960s [4]. Since then, diverse variants were born with improvements [5]-[10], concerning essentially the way of making scanning or detection. Experimental works were accompanied with analytical developments to know the shape of signal generated by defect presence and to be able to characterize it. This NDT technique by active thermography for crack in metallic materials is different from classically method used for composites, in which flash lamps are generally used as a source of excitement.

Besides, during inspections, materials can have various roughness which can generate locally absorptivity and/or emissivity changes. Furthermore, crack can also behave as a located black body. It happens while optical defect masks thermal resistance effect. In this case, Crack could be confused with regards to simple stripes, for example. To mitigate this problem, an original method was developed [8], consisting in making a round trip of laser source. This process allows then to eliminate the optical effects and to amplify the thermal defect signature. This method based on line laser excitation is used in our work to detect crack in metallic materials.

The first stage consists in feeding numerical model by a series of experimental characterizations of materials, laser and cameras, which could be used during various tries. Then, a numerical model developed with COMSOL Multuphysics ${ }^{\circledR} 5.2$ coupled with a numerically simulated camera, allows us to generate synthetic images of a thermal scene, such as a real camera would see it. The numerical model then serves as basis to realize numerical experimental designs.

\section{Principles of experimental measurements and characterizations}

\subsection{Principles of measurements}

As illustrated in Fig. 1, both configurations of measurements must be experimentally and numerically identical. Fig. 1a shows laser beam moving with a velocity $v$ following $\mathrm{x}$ direction. Temperature is measured on laser irradiated surface in the place where is located laser line thanks to a pin of thermographic detectors (in $y$ direction). In Fig. 1b, laser line moves at the same time as a test point which plays the role of detector in Comsol simulation. Temperature is registered on every time step. An interpolation is also possible to adapt time step to the frequency of the chosen camera. This point is equivalent to the signal of a pixel of a fictive camera.

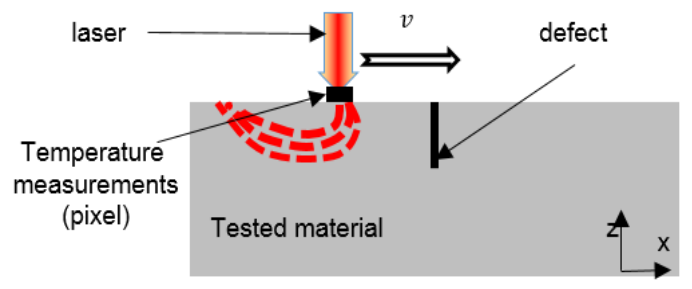

a - Side view $(x, z)$

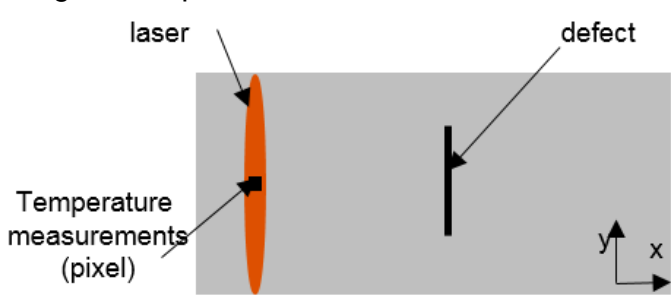

b - Upper view $(x, y)$

Fig. 1 : Principle of the method 


\subsection{1/qirt.2016.135}

\subsection{Experimental characterization}

For numerical simulation needs, validation of temperature fields and code about simulate camera, experimental characterization are obviously necessary. The first difficulty for the main part in the fact that thermal imaging is used for anomalies detection, on materials with low emissivity, high thermally diffusivity, weakly absorbent in laser radiation, and in a thermally disturbed environment, all this for low temperature variation with regard to ambient temperature. Environmental radiance temperature (reflective temperature) constitute a key parameter in these conditions, because it strongly disturbs calculated final temperature. It is evaluated according to ASTM E1862-97 recommendation.

Emissivity of material was obtained by using a classic method of comparison, thanks to a black paint of known emissivity which is close to 1 , applied to a healthy zone of the sample. Dimensions of the line laser were determined by using a plate of a strongly absorbing cover in laser radiation and highly emissive, in order to obtain the most possible contrast signal. Thermography imaging for the first moments of laser irradiation allows us, by this process to find the full width at half maximum (FWHM) up to the laser beam. Thanks to this value we can reach in by equation (1). Line laser we study has a Gaussian profile. Fig 2 gives the profile we obtained after characterization. It is reconstituted with Comsol. The laser power is also measured thanks to a power meter. Camera protection with regards to radiance requires using of a filter which was beforehand tested. Its transmission is taken into account. The absorptivity of the material is estimated at 0.32 [11]. $\sigma$ is the laser radius at $1 / \mathrm{e}$ of its amplitude.

$$
F W H M=2,355 * \sigma
$$

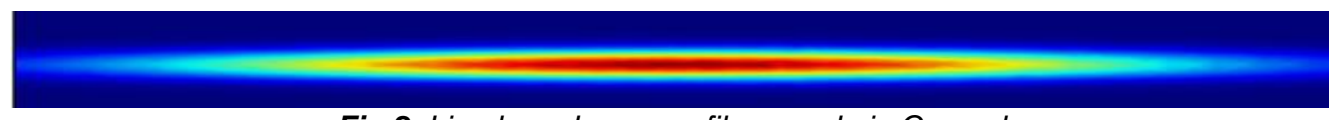

Fig 2 :Line laser beam profile remade in Comsol

\section{Numerical Modelling}

\subsection{Description}

For modeling crack detection, various approaches are used. We can note purely analytical approaches [8], [12], [13]. Other approaches were developed in order to take into account "optical" aspect of the defect [14], [15]. Nowadays finite elements methods are very wide-spread with diverse and varied approaches which concern essentially limited on punctual laser using [16], [17].

In our study, various approaches were explored:

1. The defect is filled with air and we attribute it an absorptivity different from metal one. This absorptivity is calculated thanks to Casselton formula [6].

2. The defect is filled with air and its absorptivity is equal to zero

3. We consider the defect as a thin resistive layer as illustrated in Fig 3a. Imposed thermal resistance is calculated according to the opening of studied defect and its volume.

This third approach corresponds exactly to what we are looking for experimentally. It is used in this work. With this model, we will not have meshing problem, and we reduce significantly time calculation. Fig $3 b$ shows complete geometry used for numerical simulation. Thickness is determined by taking into account thermal diffusion layer.

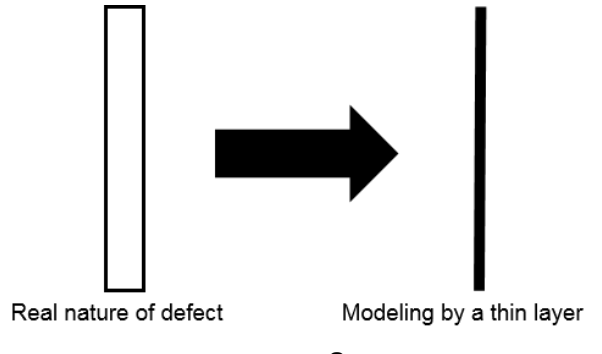

a

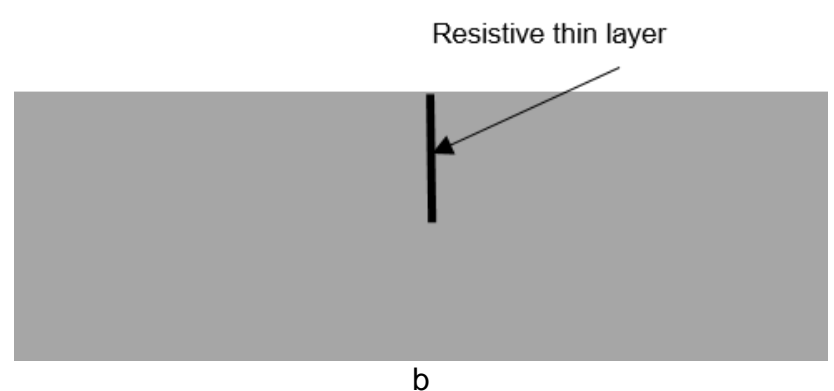

Fig 3 : Adopted geometry

\subsection{Adopted numerical modeling method}

This study is made in two main stages: 


\subsection{1/qirt.2016.135}

The first one consists in modelling the thermal scene with Comsol Multiphysics. These calculations allow us to obtain temperature fields of material surface, for every time step with a given spatial resolution. This spatial and temporal resolution must be better than the chosen camera.

The second stage consists in exporting these temperature fields in a code which we developed with Matlab allowing us to simulate thermal camera images. The aim is to take into account camera parameters (emissivity, reflected temperature, IFOV, frequency, etc...) during observation.

In this configuration, the choice of not using symmetry has been made. The obtained temperature map is used to generate thermal scene converted by the fictive camera according to the chosen parameters (IFOV, frequency, ...). Fig 4 a gives an example of geometry, Fig $4 \mathrm{~b}$ illustrates the adopted meshing.
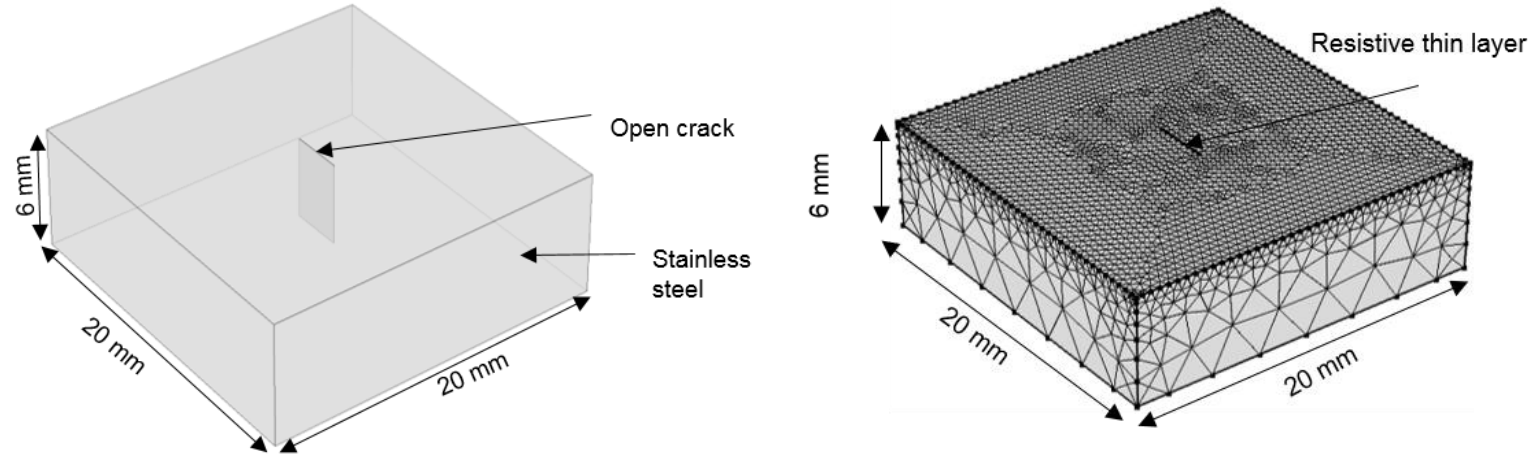

Fig 4 : Geometry and meshing of the examined material

Heat equation is used to solve this problem. Without thermal source this equation is given by Eq 2 .

$$
\frac{\partial^{2} T}{\partial x^{2}}+\frac{\partial^{2} T}{\partial y^{2}}+\frac{\partial^{2} T}{\partial z^{2}}-\frac{\rho C p}{k} \frac{\partial T}{\partial t}=0
$$

$T$ is temperature, $k$ is thermal conductivity, $C_{p}$ is the specific heat capacity, and $\rho$ is the density.

Line laser is represented by a power density which is given by Eq 3 .

$$
\varphi=A \cdot P \cdot \frac{1}{\sigma_{y} \sqrt{2 \pi}} e^{\frac{-\left(y-y_{0}\right)^{2}}{2 \sigma_{y}^{2}}} \cdot \frac{1}{\sigma_{x} \sqrt{2 \pi}} e^{\frac{-\left(x-x_{0}-v t\right)^{2}}{2 \sigma_{x}^{2}}}
$$

$\mathrm{A}$ is the absorption factor, $\mathrm{P}$ is the laser power, $\sigma_{x}$ and $\sigma_{y}$ respectively represent radius of laser at $1 / \mathrm{e}$ of amplitude on $\mathrm{x}$ and $\mathrm{y}$ axes. $\varphi$ is the power density, $v$ is the laser velocity.

\section{Results}

\subsection{Signal profile}

A defect thermal signature is visible on Fig 5 . The one-way signal presents a peak given by thermal resistance and a hollow due to a deficit of heat, where from the naming "bipolar signal". Numerically we can notice that the fact of making a round trip allows to double the obtained bipolar signal. 

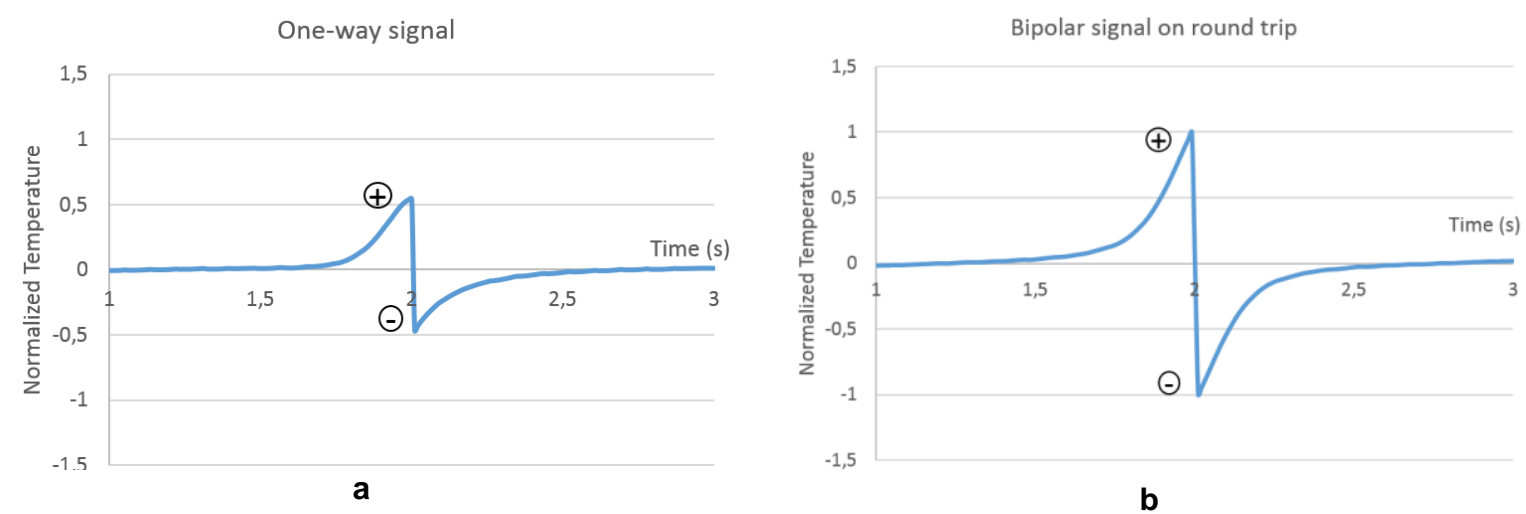

Fig 5 : Normalized temperature on one-way (a) and round trip signal (b)

\subsection{Comparison between simulation and experimental fields temperature}

The aim of this part is to compare temperature between simulation and experimental in order to validate our model. Comparison is made by using maximal rises of temperature obtained under the line laser compared with material initial temperature. We used a steel material with an open crack and the other material is a stainless steel piece without defect. Five (5) velocity (V1, V2, V3, V4, and V5; with $\mathrm{V} 1<\mathrm{V} 2<\mathrm{V} 3<\mathrm{V} 4<\mathrm{V} 5)$ were used for these measurements.

\subsubsection{Case of material without defect: stainless steel}

As illustrated in Table 1, we can notice that temperature difference between simulation and measurement are low. They are all inferiors to $1^{\circ} \mathrm{C}$. Few differences observed could come from emissivity errors, of powers, environmental temperatures etc... The relative gap with regard to temperature rise is lower than $5 \%$.

Table 1 : Temperature differences between numerical simulation and measurements in the case of stainless steel material

\begin{tabular}{|c|c|c|}
\hline Vitesse & $\begin{array}{c}\text { Temperature differences on the surface } \\
\text { of the material }\left({ }^{\circ} \mathrm{C}\right)\end{array}$ & $\begin{array}{c}\text { Relative gap with regard to temperature rise } \\
(\%)\end{array}$ \\
\hline V1 & 0,5 & 3,8 \\
\hline V3 & 0,4 & 3,4 \\
\hline V4 & 0,1 & 1,1 \\
\hline
\end{tabular}

\subsubsection{Case of a material with an open crack: steel}

We measures temperature rise on two points. On the surface of the healthy material $(A)$ and just before the defect $(B)$. This description is illustrated in Fig 6 (point $(B)$ ) gives us temperature rise due to thermal resistance. $40 \mathrm{~W}$ power with a line Gaussian laser is used for this heating.

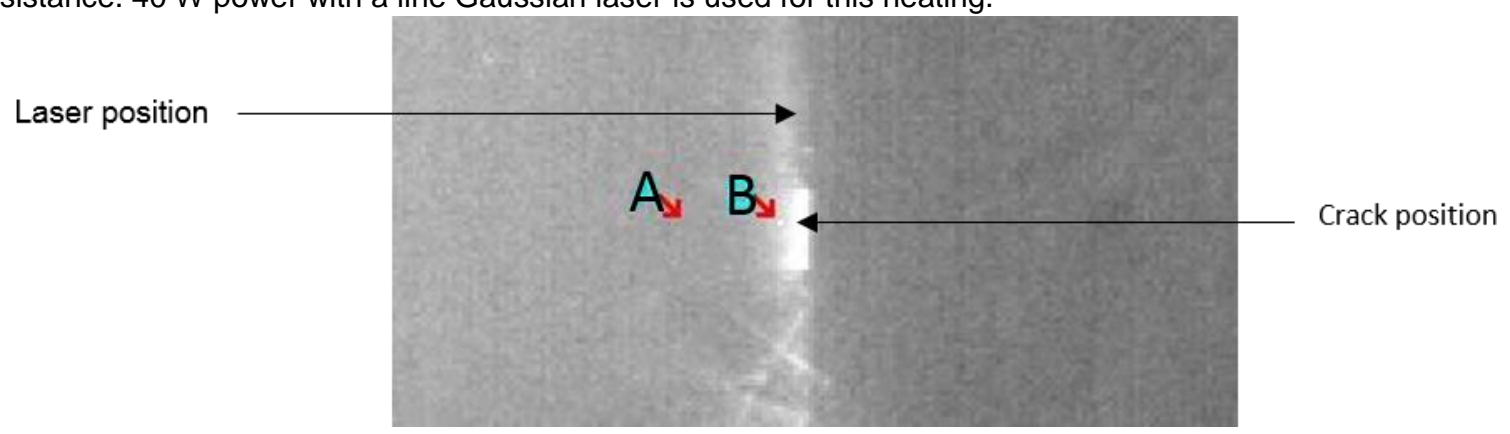

Fig 6 : Position of test points and line laser for the post-treatment

Table 2 gives temperature differences between numerical simulation and measurements with steel sample. We can notice that numerical and experimental results have the same order of magnitude far from the defect and closely to the defect (thermal resistance effect). We can also notice that amplitudes (thermal signature of the 


\subsection{1/qirt.2016.135}

defect) for numerical and experimental have the same order for the various velocity. The variances turn around $1^{\circ} \mathrm{C}$ which corresponds to $8 \%$ rise during laser passage.

Table 2 : Temperature differences between numerical simulation and measurements for a steel piece with an open crack

\begin{tabular}{|c|c|c|c|c|}
\hline Velocity & $\begin{array}{c}\text { Temperature } \\
\text { difference on the } \\
\text { surface far from the } \\
\operatorname{defect}\left({ }^{\circ} \mathrm{C}\right)\end{array}$ & $\begin{array}{c}\text { Relative gap with } \\
\text { regard to temperature } \\
\text { rise far from the defect } \\
(\%)\end{array}$ & $\begin{array}{c}\text { Temperature } \\
\text { difference near } \\
\text { the defect }\left({ }^{\circ} \mathrm{C}\right)\end{array}$ & $\begin{array}{c}\text { Relative gap with regard } \\
\text { to temperature rise near } \\
\text { the defect }(\%)\end{array}$ \\
\hline V2 & 2,3 & 17,9 & 0,7 & 4,2 \\
\hline V3 & 0,2 & 1,7 & 0,9 & 5,9 \\
\hline V5 & 1 & 10,1 & 0,4 & 3,4 \\
\hline
\end{tabular}

These various results show simulation model reability. Thus this model is used to carry out the numerical experimental designs.

\subsection{Numerical experimental designs (NED)}

In this study, we chose both essential parameters that are reduced velocity $\left(v^{*}\right)$ of the line laser (velocity divided by a reference velocity) and the reduced opening $O^{*}$ (defect opening divided by a reference opening). Two objective functions were defined: normalized amplitude defined by amplitude signal divided by a reference amplitude and "efficiency" defined by Eqs (4) et (5). The normalized amplitude is defined by Eq 5.

$$
\begin{gathered}
E f=A n \cdot \sqrt{v^{*}} \\
A n=0,914+0.0002 \cdot O^{*}-0.0185 \cdot v^{*} \\
E f=1,068+0.001 \cdot O^{*}+0,1467 \cdot v^{*}-7,1398 \cdot 10^{-7} \cdot O^{* 2}-1,4712 \cdot 10^{-6} O^{*} \cdot v^{*}-0,0038 v^{*}
\end{gathered}
$$

We can notice on Fig 7 that reduced amplitude is higher for low reduced velocity coupled with a high reduced opening. We can also observe that, whatever is the opening, the highest amplitudes are obtained with low velocity. That is due to important heat accumulation for low velocity. However, it would be necessary to adopt not too lower velocity to be effective in term of process productivity, but also not too high in order to not degrade the signal. That is in this perspective that we set up an objective function named "efficiency". Fig $7 \mathrm{~b}$ gives ranges of optimal reduced velocity which allow good detection. For example, in this studied case, optimal reduced velocity is around 18.

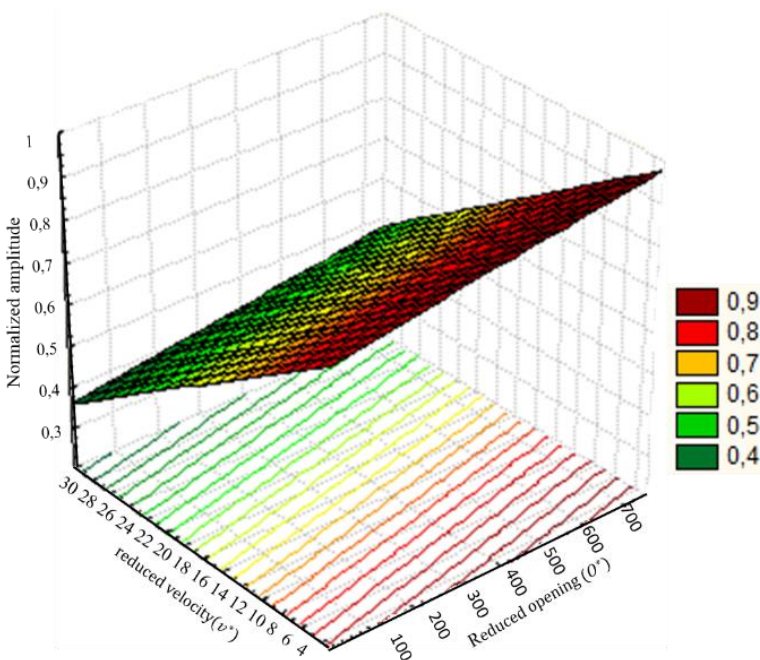

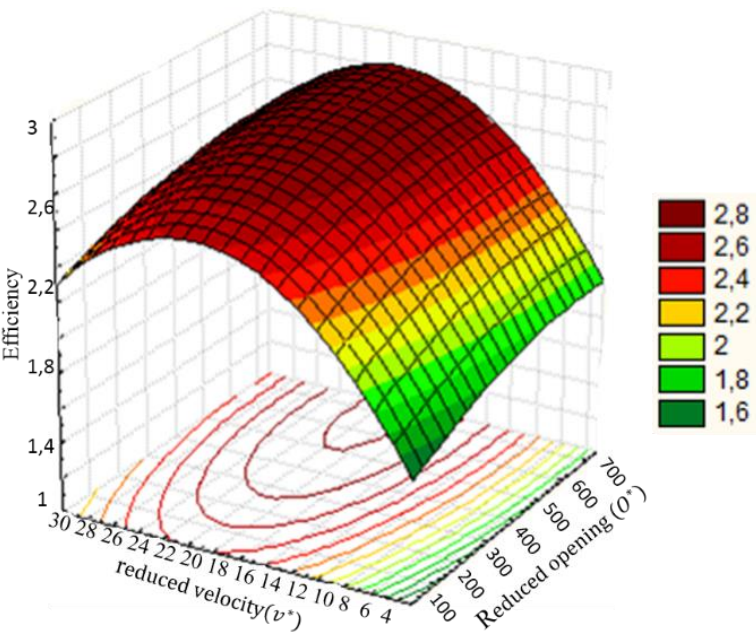

b

Fig 7 : Influence of travel velocity and opening on bipolar amplitude signal, a: normalized amplitude, b: efficiency 


\subsection{1/qirt.2016.135}

\section{Conclusion}

The realized experimental characterizations allow us to feed a simulation model by finite elements coupled to infrared thermal observation. The use of resistive thin layer is sufficient for modelling thermal signal due to with a round trip scanning. This process allows to double the defect thermal signature and to eliminate local changes of absorptivity and/or emissivity.

The realized measurements allow to see that the simulated and measured temperatures fields for a healthy material are almost identical. The calculated temperature rises due to a thin thermal resistance are the same order of magnitude than measured one. Some observed differences probably come from errors on the laser power value and the emissivity and absorptivity values.

The developed numerical model allows us to carry out the first study of the process by numerical experimental designs. This study permits to have an idea on the effects velocity and defect opening influence on the signal amplitude. It shows that most low speed, greater is the signal amplitude. It also shows that bipolar signal amplitude evolves linearly with these two parameters. The NED also allows us to determine process efficiency, which is the optimal reduce velocity in order to obtain good and rapid detection.

\section{REFERENCES}

[1] Chemin Pierre, "Ressuage," 06-Oct-2014. [Online]. Available: http://www.techniques-ingenieur.fr.proxyscd.u-bourgogne.fr/res/pdf/encyclopedia/42586210-r6201.pdf. [Accessed: 29-Mar-2016].

[2] S. Graveleau, P. Chemin, "magnétoscopie- aspects théoriques et réglementaires," 2015. [Online]. Available: http://www.techniques-ingenieur.fr.proxy-scd.u-bourgogne.fr/res/pdf/encyclopedia/42586210-r6202.pdf. [Accessed: 29-Mar-2016].

[3] G. Gaussorgues, la thermographie infrarouge. TEC \& DOC, 1999.

[4] E. J. Kubiak, "Infrared Detection of Fatigue Cracks and Other Near-Surface Defects," Appl. Opt., vol. 7, no. 9, p. 1743 , Sep. 1968.

[5] I. Kaufman, P.-T. Chang, H.-S. Hsu, W.-Y. Huang, and D.-Y. Shyong, "Photothermal radiometric detection and imaging of surface cracks," J. Nondestruct. Eval., vol. 6, no. 2, pp. 87-100, Jun. 1987.

[6] J. L. Bodnar and M. Egée, "Wear crack characterization by photothermal radiometry," Wear, vol. 196, no. 12, pp. 54-59, Aug. 1996.

[7] J. C. Krapez, D. Balageas, A. Deom, and F. Lepoutre, "Early Detection By Stimulated Infrared Thermography. Comparison With Ultrasonics and Holo/Shearography," in Advances in Signal Processing for Nondestructive Evaluation of Materials, X. P. V. Maldague, Ed. Springer Netherlands, 1994, pp. 303-321.

[8] C. Gruss, "Caméra photothermique etude théorique et réalisation pratique d'une caméra infrarouge active avec excitation laser," Université de Poitiers, 1992.

[9] S. Hermosilla-Lara, Amélioration d'une caméra photothermique par traitements d'images adaptés à la détection de fissures débouchantes. Cachan, Ecole normale supérieure, 2002.

[10] Y.Caulier, M. Taglione, "la caméra photothermique active: une technique de controle industrielle automatique en alternative au ressuage et la magnétoscopie." [Online]. Available: http://www.ndt.net/article/cofrend2014/papers/MA2C1_Y_CAULIER.pdf. [Accessed: 29-Mar-2016].

[11] Solar Absorptances and Spectral Reflectances of 12 Metals for Temperatures Ranging from 300 to $500 \mathrm{~K}$. National Aeronautics and Space Administration, 1969.

[12] R. Celorrio, A. J. Omella, A. Mendioroz, A. Oleaga, and A. Salazar, "Advances in Crack Characterization by Lock-In Infrared Thermography," Int. J. Thermophys., vol. 36, no. 5-6, pp. 1202-1207, Jul. 2014.

[13] T. Li, D. P. Almond, and D. A. S. Rees, "Crack imaging by scanning laser-line thermography and laser-spot thermography," Meas. Sci. Technol., vol. 22, no. 3, p. 035701, Mar. 2011.

[14] P. Broberg, "Surface crack detection in welds using thermography," NDT E Int., vol. 57, pp. 69-73, Jul. 2013.

[15] J.-L. Bodnar, Radiometrie photothermique appliquee a la detection et a la caracterisation de fissures. Reims, 1993.

[16] J. Schlichting, C. Maierhofer, and M. Kreutzbruck, "Crack sizing by laser excited thermography," NDT E Int., vol. 45, no. 1, pp. 133-140, Jan. 2012.

[17] V. P. Vavilov, "Modeling thermal NDT problems," Int. J. Heat Mass Transf., vol. 72, pp. 75-86, May 2014.

\section{Acknowledgments}

We would like to thanks BPI France and the Burgundy region for ATHENA project funding. We also thanks PNB for labeling the project. 